\title{
Decoupled two-dimensional superconductivity and continuous melting transitions in layered superconductors immersed in a parallel magnetic field
}

\author{
Xiao $\mathrm{Hu}$ and Masashi Tachiki \\ Computational Materials Science Center, National Institute for Materials Science, Tsukuba 305-0047, Japan
}

(Dated: May 14, 2004)

\begin{abstract}
Possible phases and the $B-T$ phase diagram of interlayer Josephson vortices induced by a magnetic field parallel to the superconducting layers are investigated by Monte Carlo simulations based on the anisotropic, frustrated XY model. While for low magnetic fields and small anisotropy parameters a single first-order transition is observed similarly to the melting of Abrikosov (or pancake) vortex lattice, an intermediate phase, characterized by decoupled, two-dimensional (2D) quasi long-range crystalline order (QLRCO) and superconductivity, is found at high magnetic fields and large anisotropy parameters. Combining the simulation results with a symmetry argument, it is revealed that this intermediate phase is of Kosterlitz-Thouless (KT) type, and the melting of 2D quasi Josephson vortex lattices and suppression of superconductivity is a KT transition. Evolution of the intermediate phase to the low-temperature phase of 3D LRCO is second order and belongs to the 3D XY universality class. The three phase boundaries merge at a multicritical point at the magnetic field of order $B_{\mathrm{mc}}=\phi_{0} / 2 \sqrt{3} \gamma d^{2}$ in the $B-T$ phase diagram. It is revealed that decoupling of the $3 \mathrm{D}$ Josephson vortex lattice into the $2 \mathrm{D}$ phase is triggered by hops of Josephson flux lines across superconducting layers activated by thermal fluctuations. The equilibrium phase diagram with the KT phase at high magnetic fields and large anisotropy parameters is consistent with the peculiar Lorentz-force-independent dissipation observed in highly anisotropic high- $T_{c}$ superconductor $\mathrm{Bi}_{2} \mathrm{Sr}_{2} \mathrm{CaCu}_{2} \mathrm{O}_{8+y}$ by Iye et al. (Physica 159C, 433 (1989)).
\end{abstract}

PACS numbers: 74.60.Ge, 74.20.De, 74.25Bt, 74.25.Dw

\section{INTRODUCTION}

The high- $T_{c}$ superconductors share a layered structure in which the superconductivity is widely believed to occur mainly in the $\mathrm{CuO}_{2}$ layers intervened by layers of charge reservoir (block layers). For many purposes, the materials can be considered as intrinsic stacks of superconducting layers, coupling with each other by the Josephson effect. They are therefore strongly anisotropic between the normal (c-axis) and in-plane directions. In spite of this anisotropy, the superconductors are threedimensional (3D) in nature at temperatures near the superconductivity transition point at zero magnetic field $T_{c}$. Especially, the critical phenomena of superconductivity transition are governed by the 3D XY universality class [1]. Since the coherence lengths diverge in the critical region, the short-range (SR) nonuniformity caused by the layer structure becomes unimportant.

There are two cases that physics can be different. The first case is realized at low temperatures, at which the correlation length $\xi_{c}$ becomes comparable with, and even smaller than the interlayer separation $d$, where twodimensional (2D) properties can arise. A crossover between $2 \mathrm{D}$ and $3 \mathrm{D}$ behaviors is expected to occur when the value of $\xi_{c} / d$ is of the order of unity. The second case is realized when the superconductor is immersed in a strong magnetic field parallel to the $\mathrm{CuO}_{2}$ layers. The coupling between the superconducting layers are weakened significantly by the magnetic field, and the effective correlation length $\xi_{c}$ can be very small even at high temperatures. To elucidate the second case is the objective of the present study. As revealed in the present paper, there is a genuine thermodynamic phase transition between 2D and 3D phases at high magnetic fields.

It is now well established that the superconductivity transition in a type II superconductor in magnetic fields [2] is first order, accompanied by the freezing of the fluxline liquid into 3D lattice [3, 4, 5]. This notion is particularly important because the transition was considered second order for a long time. We notice however that the first-order normal to superconductivity transition is mainly observed under magnetic fields perpendicular to the layers, where the 2D translation symmetry enjoyed by pancake vortices is broken at the transition. In a sharp contrast, a parallel magnetic field penetrates the layered superconductor in the form of Josephson vortex of flux quantum [6] through block layers 7]. The relevant $c$-axis translation symmetry is reduced a priori to a discrete one, raising the possibility of new phases and new melting process.

As an order competing with the 3D lattice order, decoupled superconducting layers with in-plane quasi-longrange (QLR) correlations was proposed by Efetov even before the discovery of high- $T_{c}$ superconductors with profound layer structures [8]. Later on, an exponential dependence of the interlayer shear modulus on the magnetic field is derived by Ivlev et al. [9], consistently with the decoupling scenario since the small shear modulus can hardly hold the 3D lattice when thermal fluctuations set in. This possibility was however questioned by Mikheev and Kolomeisky [10] using the renormalization group (RG) approach. It is derived that any weak interlayer coupling should be relevant and only a 3D LR crystalline order (CO) is possible, as far as hops of Joseph- 
son flux lines across $\mathrm{CuO}_{2}$ layers can be neglected, an approximation adopted in the previous works. A similar conclusion was obtained by Korshunov and Larkin 11.

Transport experiments on high- $T_{c}$ superconductors have been sheding lights on the possible crystalline order of Josephson flux lines. A peculiar Lorentz-force-independent dissipation has been found in $\mathrm{Bi}_{2} \mathrm{Sr}_{2} \mathrm{CaCu}_{2} \mathrm{O}_{8+y}$ under $H=5 T$ by Iye $e t$ al. that the resistivity is independent of the angle between the magnetic field and current when they are both parallel to the $\mathrm{CuO}_{2}$ layer 12]. In the same family of materials, non-Ohmic power-law I-V characteristics are observed by other groups 13, 14]. Chakravarty et al. demonstrated that the Lorentz-force-independent dissipation cannot occur in a lattice phase [15]. In order to explain these peculiar dissipations, Blatter et al. proposed a KosterlitzThouless (KT) transition 16, 17] at high magnetic fields, relating the creeping of Josephson flux lines across the $\mathrm{CuO}_{2}$ layers caused by applied currents with the shearless flux-line state [18]. (See 19] for a possible KT phase at intermediate magnetic fields.) On the other hand, an excess resistivity for $H \perp I$ over $H \| I$, and thus a Lorentz-force-dependent dissipation was observed in $\mathrm{YBa}_{2} \mathrm{Cu}_{3} \mathrm{O}_{7-\delta}$ single crystals at low fields [20, 21, 22]. Kes et al. addressed this difference in terms of the different anisotropy parameters [23]. Taking the Bi-based materials almost "magnetically transparent", the authors attributed the experimentally observed dissipations to uncontrolled miss alignments of magnetic field.

Motivated by an experiment by Kwok et al. suggestive of continuous melting transition at intermediate magnetic fields 24], Balents and Nelson proposed a smectic phase: a regular subset of block layers are selectively occupied by Josephson vortices; in the occupied block layers Josephson flux lines behave like liquid. They showed that melting of the 3D lattice can be continuous in the presence of layer pinning [25]. See also [26].

Although these studies provide important physical insights for the mixed states in layered type II superconductors, it is clear that discrepancies among different approaches have not been resolved and that a unified picture is still not available. The main difficulty lies in the features of different energy contributions involved in the present system: First, the inter-vortex repulsive force is highly anisotropic between the $c$ axis and the $a b$ plane; Second, Josephson flux lines feel strong pinning potentials from the $\mathrm{CuO}_{2}$ layers. At a first glance, approximations which take into account differences in strengths of these energy contributions can simplify the problem. It turns out that the situation is quite complicated. The Josephson flux lines arrange themselves such that they reside at positions from which deviations in different directions cost equal energies. Although the profound layer structure reduces the $c$-axis component of the displacement field at low temperatures, it certainly enhances the rigidity of the flux-line lattice and stabilizes it to high temperatures. At the melting temperature, thermal fluctuations are significant and smear out the strong layer pinning to an effectively weak one. Therefore, no approximation can be justified easily in the regime of phase transition. One thus has to treat the competition among the anisotropic repulsion energy, the periodic layer pinning potential, and thermal fluctuations simultaneously.

On the other hand, since a Josephson vortex is of large size in the in-plane direction, point-like defects play much less pinning effect compared with a pancake vortex. It relieves one from being involved into glassy problems, and makes the study on a pure Josephson-vortex system more relevant to reality. As a result, the high-field part of the $B-T$ phase diagram for a pure vortex system becomes accessible experimentally. For this point, please see several interesting proposals [27, 28, 29] in terms of pancake vortices, which are however hampered by inevitable point-like defects in superconductors which govern the behaviors of pancake vortices.

These situations motivate us to investigate Josephsonvortex systems by means of Monte Carlo simulations. The Hamiltonian is the so-called anisotropic, frustrated $\mathrm{XY}$ model on the superconductivity order parameter, where the frustrations in phase variables are induced by the magnetic field. This model has been used to simulate quite successfully the melting phenomenon of Abrikosov (or pancake) vortex lattice under magnetic fields parallel to the $c$ axis $30,31,32,33,34,351$. We adopt the same model in the present study on interlayer Josephson vortices. The main results are summarized as follows. There is a multicritical point in the $B-T$ phase diagram: below the multicritical field a single first-order melting transition upon temperature sweeping is observed; above it there exists an intermediate $\mathrm{KT}$ phase characterized by in-plane 2D, QLRCO and superconductivity in between the normal phase and 3D lattice phase, accompanied by two continuous melting transitions. The existence of a KT phase at magnetic fields above the multicritical point explains the peculiar dissipations, such as the Lorentz-force-independent resistance and the powerlaw non-Ohmic I-V characteristics, observed in Bi-based materials for which the multicritical magnetic field is approximately $B_{\mathrm{mc}} \simeq 2 T$ presuming $\gamma=150$. In contrast, Lorentz-force-dependent dissipations are expected for $\mathrm{YBa}_{2} \mathrm{Cu}_{3} \mathrm{O}_{7-\delta}$, since to access the $\mathrm{KT}$ phase one needs a magnetic field above $B_{\mathrm{mc}} \simeq 50 T$ taking $\gamma=8$. Some of the results were published in Ref. 36, 37.

The remaining part of this paper is organized as follows. In Sec. II the model Hamiltonian is introduced and some details of the simulation techniques are presented. After a discussion on the description on flux line lattice, we show in Sec. III simulation results on the first-order melting for a system corresponding to $\mathrm{YBa}_{2} \mathrm{Cu}_{3} \mathrm{O}_{7-\delta}$ single crystals. It then follows numerical evidences on an intermediate phase in a highly anisotropic system. Detailed characteristics are provided which allow us to conclude the KT nature of this phase. The superconductivity transition is discussed in Sec. IV, and the relationship with the crystallin order of Josephson vortices is revealed. Section V addresses the possible universality 
of the transition between 2D phase and the 3D lattice. The $B-T$ phase diagram of Josephson vortex systems is mapped out in Sec. VI. Finally, summary on the main results derived from simulations and discussions on recent experiments are given in Sec. VII.

\section{MODEL AND SIMULATION DETAILS}

In the presence of magnetic field, the amplitude of superconductivity order parameter, associated with the local tendency of electron pairing, attains a finite value at $H_{c 2}$ through a crossover. The genuine thermodynamic phase transition takes place at a temperature lower than that corresponding to $H_{c 2}(T)$. Therefore, the most important thermal fluctuations in the thermodynamic phase transition come from phase variables of the superconductivity order parameter. Under a magnetic field parallel to the layers, the effective Hamiltonian is thus 37.

$$
\begin{aligned}
& \mathcal{H}=-J \sum_{\mathbf{R}_{i}-\mathbf{R}_{j} \| x, y \text { axis }} \cos \left(\varphi_{i}-\varphi_{j}\right) \\
& -\frac{J}{\gamma^{2}} \sum_{\mathbf{R}_{i}-\mathbf{R}_{j} \| c \text { axis }} \cos \left(\varphi_{i}-\varphi_{j}-\frac{2 \pi}{\phi_{0}} \int_{\mathbf{R}_{i}}^{\mathbf{R}_{j}} \mathbf{A} \cdot d \mathbf{R}\right) .
\end{aligned}
$$

The model is defined on the simple cubic grid with the unit length equal to the separation between $\mathrm{CuO}_{2}$ layers $d$. The couplings given by $J=\phi_{0}^{2} d / 16 \pi^{3} \lambda_{a b}^{2}$ are limited to nearest neighboring grid sites and $\gamma=\lambda_{c} / \lambda_{a b}$. To be specific, we put $\hat{x} \perp \hat{y} \perp \hat{c}$ and $\mathbf{B} \| \hat{y}$, and choose the Landau gauge $\mathbf{A}=(0,0,-x B)$ in Eq.(1).

The cosine functions of the gauge invariant phase differences in $a b$ planes model the kinetic energy terms in a Ginzburg-Landau free energy functional. Higher harmonics are neglected since the lowest order terms with $2 \pi$ modulation are sufficient for describing relevant thermal fluctuations at phase transitions. The second term in the Hamiltonian is the Josephson energy between neighboring $\mathrm{CuO}_{2}$ layers. The magnetic field weakens the interlayer coupling by inducing frustrations. Our Hamiltonian can be derived from the Lawrence-Doniach free energy functional [38], which is proposed for layered superconductors and recognized as a good model for high- $T_{c}$ superconductors with profound layer structure.

Spatial variations and thermal fluctuations in the magnetic induction, and thus magnetic couplings, are neglected in Hamiltonian (1), which is in principle justified only at the strongly type II limit. It is a reasonable approximation in the present case. The London penetration depth $\lambda_{c}$ is very large (of order $\mu m$ ), which makes the spatial variation of the magnetic induction in the $x$ direction and thus the magnetic interactions among Josephson flux lines very small. Magnetic couplings may play a role when pancake vortices are activated by thermal fluctuations. It was addressed, however, that the magnetic interaction between two pancake vortices on a same $\mathrm{CuO}_{2}$ layer increases with the separation only as $1 / R$ at large distance because of the interlayer coupling [18], which is therefore much smaller than the elastic energy. Therefore, magnetic couplings can be ignored safely as far as the thermodynamic phase transition is concerned.

Thermal fluctuations in the amplitude of superconductivity order parameter can be taken into account in several ways. One can include a temperature dependence of the amplitude in the mean-field way, such that the penetration depth and the coupling constants vary with temperature. This treatment permits a quantitative comparison between simulated quantities and experimental observations [32, 39]. Alternatively, one can take thermal averages of superconductivity order parameter on scales larger than the unit length of grid in $a b$ planes. A fully disordered configuration of phase variables on the scale of $d$ then results in zero superconductivity order parameter at any larger length scales.

The Hamiltonian (1) provides a reasonable description for the layer pinning on Josephson vortices in the whole temperature regime, even without taking into account the temperature dependence of Ginzburg-Landau correlation length. At low temperatures, the layers modeled by the discreteness of the underlying grid in the $c$ direction set a series of barriers to the motion of Josephon flux lines. If temperature is high enough, on the other hand, pancake vortices are activated thermally on $\mathrm{CuO}_{2}$ layers, such that Josephson flux lines can hop to neighboring block layers easily. Thermal fluctuations reduce effectively the layer pinning potential.

The above Hamiltonian has a trivial limit $\gamma=\infty$, where $\mathrm{KT}$ transition takes place at $T_{\mathrm{KT}}^{\mathrm{b} a r e} \simeq 0.89 \mathrm{~J} / k_{B}$ in each independent $a b$ plane. For large but finite anisotropy parameters, there is a broad crossover regime which is dominated by this limit, which can be seen in the specific heat [37, 40].

In the present study, the magnetic field is fixed at $f \equiv B d^{2} / \phi_{0}=1 / 32$ while the anisotropy parameter is tuned for the convenience of simulation. The system size is $L_{x} \times L_{y} \times L_{c}=384 d \times 200 d \times 20 d$ under periodic boundary conditions. There are totally $N_{v}=48000$ Josephson vortices in the ground state. The system size is set anisotropically since we are interested in systems of large anisotropy parameters: there are $f \times L_{x} / d=12$ Josephson flux lines the $x$ direction, which is comparable with the number of Josephson flux lines in the $c$ axis: $L_{c} / 2 d=10$. (See Fig. 2 of [37].) As the field direction coincides with $a b$ planes of strong couplings, the system size in this direction has to be taken large enough in order to treat thermal fluctuations sufficiently. Although a full analysis on finite-size effects is still difficult with the available computing resource, it will be revealed later by comparing simulation results for different anisotropy parameters that the phases and phase diagram derived in the present paper are free of serious finite-size effects.

In a typical simulation process, we start with a random configuration of phase variables at a sufficient high temperature. The system is then cooled down gradually with 
the temperature skips and the number of Monte Carlo sweeps listed in Ref. 37]. After arriving at the lattice phase at a sufficiently low temperature, $T=0.1 \mathrm{~J} / k_{B}$, we heat the system back slowly. Thermal averages are taken over $\sim 10^{7}$ Monte Carlo sweeps at temperatures around the transition points. All the results shown in the present paper are calculated in the heating process.

\section{CRYSTALLIZATION TRANSITION}

\section{A. Description of Josephson vortex lattice}

The crystalline order of the system is described by the correlation function

$$
S(\mathbf{R})=\langle\rho(\mathbf{R}) \rho(0)\rangle-\langle\rho(\mathbf{R})\rangle\langle\rho(0)\rangle
$$

where $\mathbf{R}=(x, y, z)$ and $\rho(\mathbf{R})$ is the $y$ component of the vorticity at position $\mathbf{R}$ which is explicitely defined by $\rho(\mathbf{R}) \equiv \sum_{\alpha} \delta\left(\mathbf{R}_{\perp}-\mathbf{R}_{\perp, \alpha}(y)\right)$ with $\mathbf{R}_{\perp}=(x, z)$, and its Fourier transformation, i.e. the structure factor,

$$
S(\mathbf{k})=\int d^{3} R e^{-i \mathbf{k} \cdot \mathbf{R}} S(\mathbf{R})
$$

In lattice phase subject to thermal fluctuations, the vortex density is expressed in terms of the reciprocal vectors as

$\rho(\mathbf{R})=\Psi_{0} e^{-i \mathbf{K}_{0} \cdot \mathbf{R}}+\Psi_{1}(\mathbf{R}) e^{-i \mathbf{K}_{1} \cdot \mathbf{R}}+\Psi_{2}(\mathbf{R}) e^{-i \mathbf{K}_{2} \cdot \mathbf{R}}+$ c.c.,

where $\mathbf{K}_{j}$ with $j=0,1,2$ are primitive reciprocal lattice vectors and $\mathbf{K}_{0}+\mathbf{K}_{1}+\mathbf{K}_{2}=0$. Higher harmonics are not included for simplicity, which are not important for the later discussions on phase transitions. $\Psi_{j}(\mathbf{R})$ 's are order parameters of the crystalline order, and are expressed by the displacement field $\mathbf{u}=\left(u_{x}, 0, u_{c}\right)$ [41]:

$$
\Psi_{j}(\mathbf{R})=e^{i \mathbf{K}_{j} \cdot \mathbf{u}(\mathbf{R})}
$$

The density-density correlation is then given by correlation functions of order parameters

$$
\begin{aligned}
S(\mathbf{R}) & =\sum_{j}\left[e^{-i \mathbf{K}_{j} \cdot \mathbf{R}}\left\langle\Psi_{j}(\mathbf{R}) \Psi_{j}^{*}(0)\right\rangle+\text { c.c. }\right] \\
& =\sum_{j}\left|\left\langle\Psi_{j}\right\rangle\right|^{2}\left[e^{-i \mathbf{K}_{j} \cdot \mathbf{R}}+\text { c.c. }\right] g\left(\mathbf{K}_{j}, \mathbf{R}\right),
\end{aligned}
$$

with the correlator of displacement fields defined by

$$
g(\mathbf{K}, \mathbf{R}) \equiv\left\langle e^{-i \mathbf{K} \cdot[\mathbf{u}(\mathbf{R})-\mathbf{u}(0)]}\right\rangle .
$$

The cross terms between different reciprocal vectors disappear because of the infinite expectation values of displacement fields. The structure factor is then given by

$S(\mathbf{k}) \sim \sum_{j} \int d^{3} R\left[e^{-i\left(\mathbf{k}-\mathbf{K}_{j}\right) \cdot \mathbf{R}}+e^{-i\left(\mathbf{k}+\mathbf{K}_{j}\right) \cdot \mathbf{R}}\right] g\left(\mathbf{K}_{j}, \mathbf{R}\right)$.

For the present flux line system, it is convenient to consider partial structure factors in the section perpendicular to the magnetic field. They are related to the above $3 \mathrm{D}$ one by a partial integral, $S\left(k_{x}, y, k_{c}\right) \sim$ $\int d k_{y} e^{i k_{y} y} S(\mathbf{k})$. In the present paper, we will show simulation results on $S\left(k_{x}, k_{c}\right) \equiv S\left(k_{x}, y=0, k_{c}\right)$ which reveals the vortex correlations in the same crosssection perpendicular to the magnetic field.

In a $3 \mathrm{D}$ crystal phase, $g\left(\mathbf{K}_{j}, \mathbf{R}\right)$ 's approach to constants at large distances, and $\delta$-function Bragg peaks should be observed at the reciprocal-lattice vectors. For the present system of weak interlayer couplings, there is a competing order to the 3D crystalline order, in which the Josephson vortices are strongly correlated in each block layer such that a QLRO is present, while only SR correlations are realized in the $c$ direction:

$$
g\left(\mathbf{K}_{j}, \mathbf{R}\right) \sim e^{-|z| / \xi_{c}} / r^{\eta}
$$

with $\mathbf{R}=(\mathbf{r}, z)$, and $\mathbf{r}$ an in-plane positional vector rescaled from the original one according to the inplane elastic constants [10, 25]. As will be revealed later, this case occurs when the mangeic field and the anisotropy parameter are large enough, such that every block layer is occupied by Josephson vortices and the two unit vectors in real space are $\mathbf{a}_{1}=(d / f, 0,0)$ and $\mathbf{a}_{2}=(d / 2 f, 0, d)$. The primitive reciprocal lattice vectors are $\mathbf{K}=( \pm 2 f \pi / d, 0, \pm \pi / d)$ and $(0,0, \pm 2 \pi / d)$. The last two ones are equivalent to the origin. In this case, dominant thermal fluctuations are associated with the other four reciprocal vectors. Especially, the $k_{c}$ and $k_{x}$ profiles of the partial structure factor with $y=0$ around them are evaluated as

$$
\begin{aligned}
S\left( \pm 2 f \pi / d, k_{c}\right) & \sim \int d x d z e^{-i\left(k_{c} \pm \pi / d\right) z} g(\mathbf{K}, \mathbf{R}) \\
& \sim \frac{\xi_{c}^{-1}}{\xi_{c}^{-2}+\left(k_{c} \pm \pi / d\right)^{2}} \\
S\left(k_{x}, \pm \pi / d\right) & \sim \int d x d z e^{-i\left(k_{x} \pm 2 f \pi / d\right) x} g(\mathbf{K}, \mathbf{R}) \\
& \sim\left|k_{x} \pm 2 f \pi / d\right|^{-1+\eta}
\end{aligned}
$$

with $\mathbf{R}=(x, 0, z)$, up to multiplicative coefficients and linear corrections. Namely, in such a phase of 2D QLRO the structure factor should show power-law singularities in the $k_{x}$ direction at reciprocal-lattice vectors, while smears out in the $k_{c}$ direction in a Lorentzian form. By fitting the profiles of the Bragg spots, the correlation 
length $\xi_{c}$ and the exponent $\eta$ of QLRO in $a b$ planes can be evaluated. Detailed discussions on profiles of Bragg peaks in the smectic phase of liquid crystals can be found in Ref.[42].

\section{B. First-order melting transition: $\gamma=8$}

Let us start with a system of the anisotropy parameter $\gamma=8$, which models the high- $T_{c}$ superconductor $\mathrm{YBa}_{2} \mathrm{Cu}_{3} \mathrm{O}_{7-\delta}$. Upon temperature sweeping, a firstorder transition is observed, indicated by the $\delta$-function peak in the specific heat and a kink anomaly in the Josephson energy at $T_{m}=0.96 \mathrm{~J} / k_{B}$, as displayed in Figs.1 and 4 of Ref. 37 .

In order to characterize the phase transition better, we investigate the crystalline order of Josephson vortices in terms of the structure factors. At low temperatures, there is a fine $3 \mathrm{D}$ LRCO in the system indicated by the $\delta$-function Bragg peaks at the reciprocal-lattice vectors, not only the minimal ones but also at higher-order satellites, as displayed in the top-left panel of Fig 1 The Bragg peaks are observed up to $T_{m} \simeq 0.96 \mathrm{~J} / k_{B}$, without losing their sharpness as shown in the top-right panel of Fig[1]and in Fig[2] The 3D LRCO disappears when temperature crosses $T_{m}$. The first-order transition at $T_{m}$ is therefore identified as the melting of the Josephson vortex lattice, similar to the melting of Abrikosov (or pancake) vortex lattice in magnetic fields parallel to the $c$ axis.

At $T \simeq 0.98 J / k_{B}$, SR correlations are observed as shown in the bottom-left panel of Fig 1 which characterize a vortex liquid with layer modulation. It is worthy to notice that, in spite of the high anisotropy in the vortex lattice, thermal fluctuations of large wave-lengths are quite isotropic for $\gamma=8$ as reflected in the spots at $\left(k_{x}, k_{c}\right)=( \pm 2 f \pi / d, \pm \pi / d)$ in the bottom-left panel of Fig 1 At higher temperatures, $T \geq 1.2 \mathrm{~J} / k_{B}$, correlations are suppressed by thermal fluctuations to the scale of grid size of simulation as depicted in the bottom-right panel of Fig 1 Containing huge number of closed vortex loops the system is completely disordered. In such a case, a coarse-graining in the $a b$ planes results in zero amplitude of the superconductivity order parameter, corresponding to the normal states. In this temperature regime vortices are therefore not suitable for describing the system anymore. The transformation from the vortex liquid to the normal phase is a crossover, characterized by the broad peak in the specific heat in Fig. 3 of Ref. [37].

We have also simulated systems with $1 \leq \gamma<8$ at $f=1 / 32$. The melting point increases with decreasing anisotropy parameter. Josephson vortices distribute in a subset of block layers for $\gamma<5$ [37]. The phase transitions are first order at which 3D LRCO sets up for all the cases we have searched. These simulation results look inconsistent with scenarios of continuous melting at intermediate and low magnetic fields.
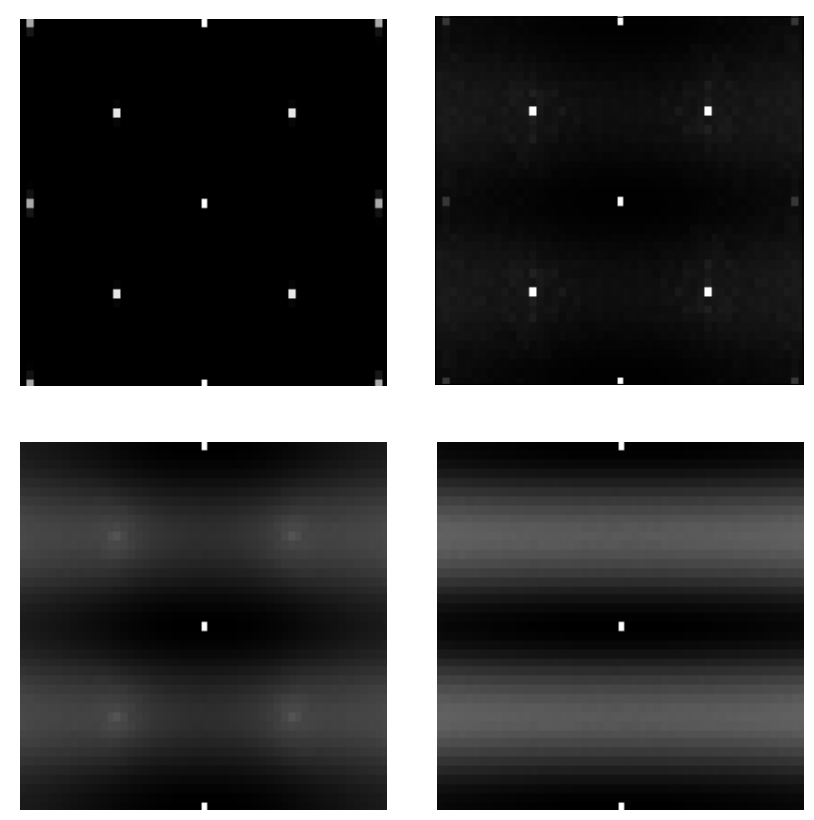

FIG. 1: Structure factors $S\left(k_{x}, k_{c}\right)$ for $\gamma=8$ at several typical temperatures. Top-left: $T=0.1 J / k_{B}$; Top-right: $T=0.96 \mathrm{~J} / k_{B}$; Bottom-left: $T=0.98 \mathrm{~J} / k_{B}$; Bottom-right: $T=1.5 \mathrm{~J} / k_{B}$. The panels are for wave numbers within $k_{x} \in$ $[-25 \pi / 192 d, 25 \pi / 192 d]$ (horizontal) and $k_{c} \in[-2 \pi / d, 2 \pi / d]$ (vertical). The spots at $\left(k_{x}, k_{c}\right)=(0, \pm 2 \pi / d)$ are quivalent to $(0,0)$ in the present system.
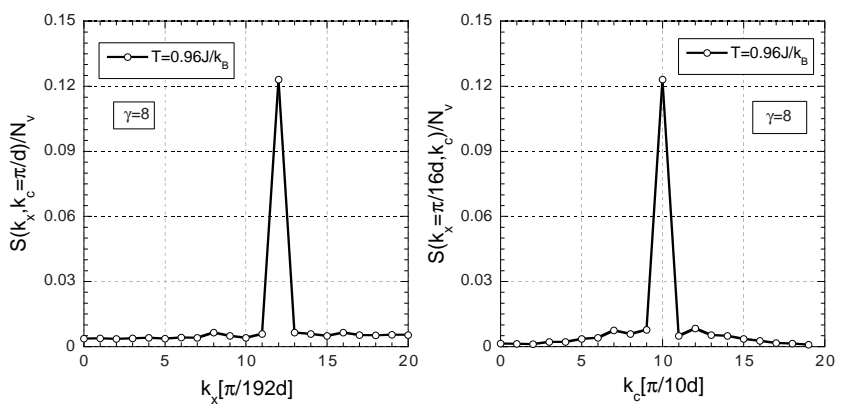

FIG. 2: $k_{x}$ (left) and $k_{c}$ (right) profiles of Bragg peaks in the top-right panel of Fig 1 for $\gamma=8$ at $T=0.96 J / k_{B}$.

\section{Multicritical point}

The first-order melting of Josephson vortex lattice is suppressed to continuous transitions when the anisotropy parameter increases to $\gamma=10$, when the filling factor is fixed at $f=1 / 32$, as reported in Ref. [37]. A critical value of the product between the anisotropy parameter and filling factor may be given by [18, 37]:

$$
f \gamma=\frac{1}{2 \sqrt{3}}
$$

It is easy to see that below the critical value, the Josephson vortex system is physically equivalent to a $3 \mathrm{D}$ 
anisotropic Abrikosov vortex lattice. Rescaling the system by the anisotropy parameter should result in an effective isotropic system. All physical properties, including possible phases and the nature of phase transition, should be essentially the same as the isotropic system except for an anisotropy scaling 43]. This physical discussion is consistent with our observations presented in the preceding subsection.

On the other hand, the system becomes un-rescalable above the critical value, since the minimal inter-vortex separation in the $c$ axis $2 d$ has been reached at the critical value. Any increase in either $f$ or $\gamma$ results in overwhelming $x$-direction inter-vortex repulsions, which enhance the in-plane crystalline order. Although the $\mathrm{CuO}_{2}$ layers fix the inter-vortex distance for Josephson vortices in the $c$ axis, it has nothing to do with the inter-vortex correlations. This is the most important peculiarity of the interlayer Josephson vortices compared with liquid crystals in which smectic orders are realized.

\section{Intermediate phase: $\gamma=20$}

In order to reveal the crystalline order of Josephson vortices above the critical point, we choose $\gamma=20$ which is quite above the multicritical value $\gamma=16 / \sqrt{3} \simeq 9.24$ at $f=1 / 32$. An appropriately large anisotropy parameter realizes the intermediate phase in a wide temperature regime while leaves a reasonable interlayer Josephson coupling, and thus is convenient for computer simulations. For $T \leq 0.65 J / k_{B}$, a $3 \mathrm{D}$ LRCO same as that for $\gamma=8$ is observed in this highly anisotropic case as shown in the top-left panel of Fig[3] and in Fig 4

In a sharp contrast to the case of $\gamma=8$, however, the Bragg spots for $\gamma=20$ at $\left(k_{x}, k_{c}\right)=( \pm 2 f \pi / d, \pm \pi / d)$ smear significantly in the $k_{c}$ direction as temperature increases to $T=0.7 \mathrm{~J} / k_{B}$, while leaving the sharpness in the $k_{x}$ direction almost unchanged as seen in the topright panel of Fig 3] Thermal fluctuations are therefore very anisotropic as well as the structure of the vortex lattice. The diffusive and stripe-like Bragg spots survive to $T \simeq 0.95 J / k_{B}$ (see the bottom-left panel of Fig 3 ).

As shown in the right panel of Fig [5] the $k_{c}$ profile of the Bragg spots at $T=0.7 \mathrm{~J} / k_{B}$ is fitted well by the Lorentzian function in Eq.10 with the correlation length $\xi_{c} \simeq 1.5 d$. Therefore, the Josephson vortices are coupled to each other only SR in the $c$ axis. Although it is not easy to distinguish numerically the $k_{x}$ profile in the left panel of Fig 5 from a $\delta$-function Bragg peak, it is easy to see that a $\delta$-function one is impossible as far as the system is decoupled in the $c$ axis.

The $k_{x}$ profiles of structure factors $S\left(k_{x}, k_{c}\right)$ for higher temperatures are depicted in Fig[6] They are clearly different from the $\delta$-function peak for $T \leq 0.65 J / k_{B}$ for the present system, and those for $\gamma=8$ in the whole temperature regime $T \leq T_{m}$. Supposing a cusp singularity in the profiles in Fig [6] we can evaluate the exponent $\eta$ in the correlation function Eq. (9) by fitting the data
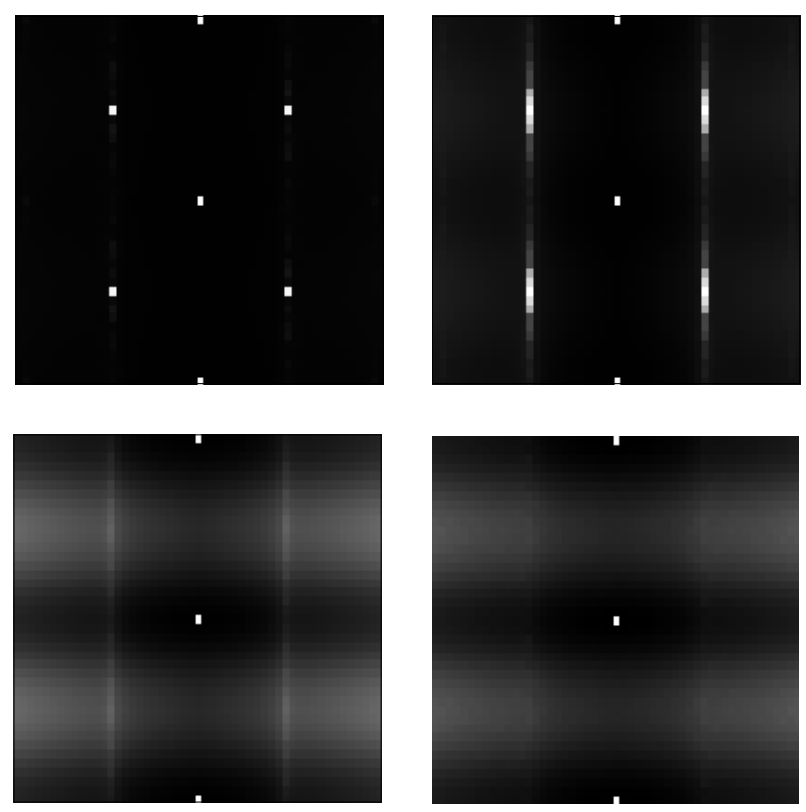

FIG. 3: Structure factors $S\left(k_{x}, k_{c}\right)$ for $\gamma=20$ at several typical temperatures. Top-left: $T=0.65 J / k_{B}$; Top-right: $T=0.7 \mathrm{~J} / k_{B}$; Bottom-left: $T=0.95 \mathrm{~J} / k_{B}$; Bottom-right: $T=0.97 J / k_{B}$. The panels are for wave numbers within $k_{x} \in$ $[-25 \pi / 192 d, 25 \pi / 192 d]$ (horizontal) and $k_{c} \in[-2 \pi / d, 2 \pi / d]$ (vertical). The spots at $\left(k_{x}, k_{c}\right)=(0, \pm 2 \pi / d)$ are quivalent to $(0,0)$ in the present system.
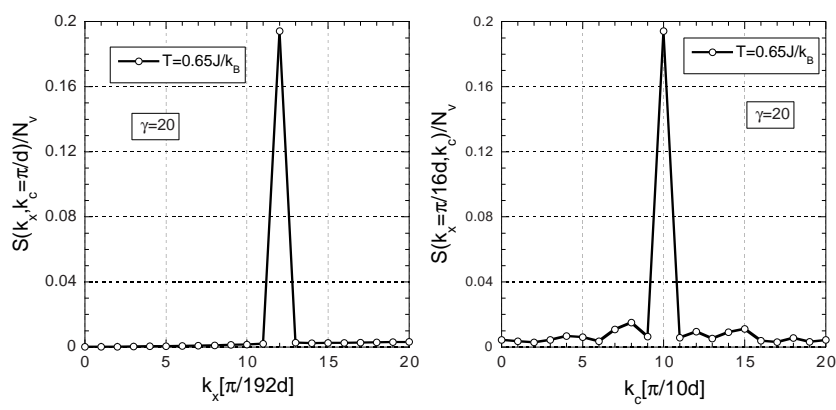

FIG. 4: $k_{x}$ (left) and $k_{c}$ (right) profiles of Bragg peaks in the top-left panel of Fig[3 for $\gamma=20$ at $T=0.65 J / k_{B}$.
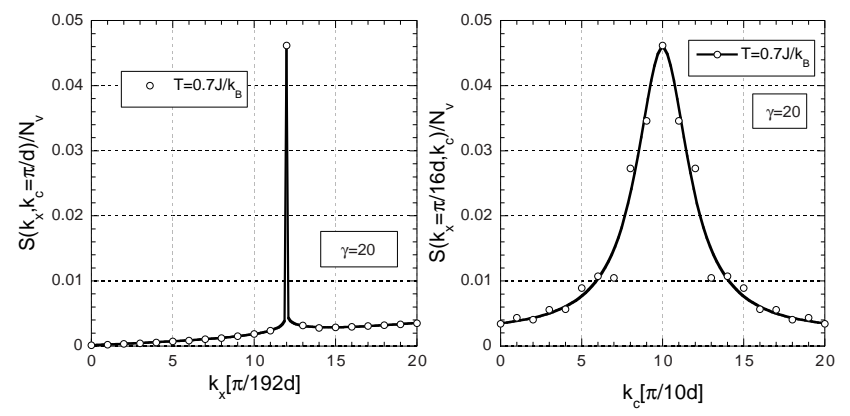

FIG. 5: $k_{x}$ (left) and $k_{c}$ (right) profiles of Bragg peaks in the top-right panel of Fig 3 for $\gamma=20$ at $T=0.7 \mathrm{~J} / k_{B}$. 


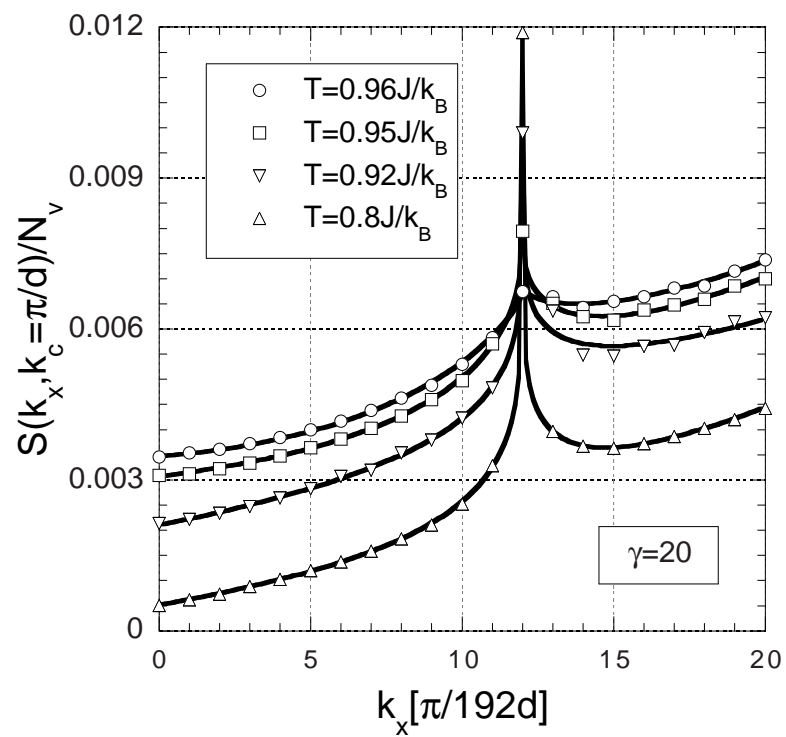

FIG. 6: $k_{x}$ profiles of structrure factors $S\left(k_{x}, k_{c}\right)$ around the Bragg spots for $\gamma=20$ in the intermediate phase. The solid curves are results of the least-squares fittings to the power-law function as described in text.

points including the peak values. This analysis results in $\eta \simeq 1.97 \pm 0.07,1.42 \pm 0.01,1.17 \pm 0.007,1.11 \pm 0.003$ at $T=0.96,0.95,0.92,0.8 \mathrm{~J} / k_{B}$, with the error bars from the least-squares fittings. The fitting curves are shown in Fig 6 by the solid curves. The good quality of the fittings with $\eta>1$ for all the temperatures, especially for $0 \leq k_{x} \leq \pi / 16 d$, can be taken as a justification of the assumption on the cusp singularity.

The temperature dependence of the maximal value of Bragg peaks is shown in Fig 7 The intensity of Bragg spots is small and increases very slowly with decreasing temperature for $0.7 \mathrm{~J} / k_{B} \leq T \leq 0.95 \mathrm{~J} / k_{B}$. Across $T_{\times}$where the spots become $\delta$-function like, the intensity increases noticably. As a comparison, that for $\gamma=8$ increases very sharply with decreasing temperature as soon as they become observable at the melting point $T_{m}$ as shown in Fig[7]

One can also evaluate the partial structure factor $S\left(k_{x}, k_{y}\right) \equiv S\left(k_{x}, k_{y}, z=0\right)$, which describes the Josephson vortex correlations in the same block layers. The profiles around the Bragg spots $\left(k_{x}, k_{y}\right)=( \pm 2 f \pi / d, 0)$ should behave as

$S\left(k_{x}, 0\right) \sim\left|k_{x} \pm 2 f \pi / d\right|^{-2+\eta}, \quad S\left(2 f \pi / d, k_{y}\right) \sim\left|k_{y}\right|^{-2+\eta}$.

The simulated results are displayed in $\operatorname{Fig} 8$ for $T=$ $0.8 J / k_{B}$. A power-law singularity is observed, which is consistent with that in the $x-c$ crosssections. The collapse of two profiles left to the peak in Fig 8 indicates that the in-plane correlation functions are governed by the same exponent $\eta$. The deviation on the right side of the peak is obviously caused by the second peak in the $k_{x}$ profile at $k_{x}=4 f \pi / d$, which is absent in the $k_{y}$

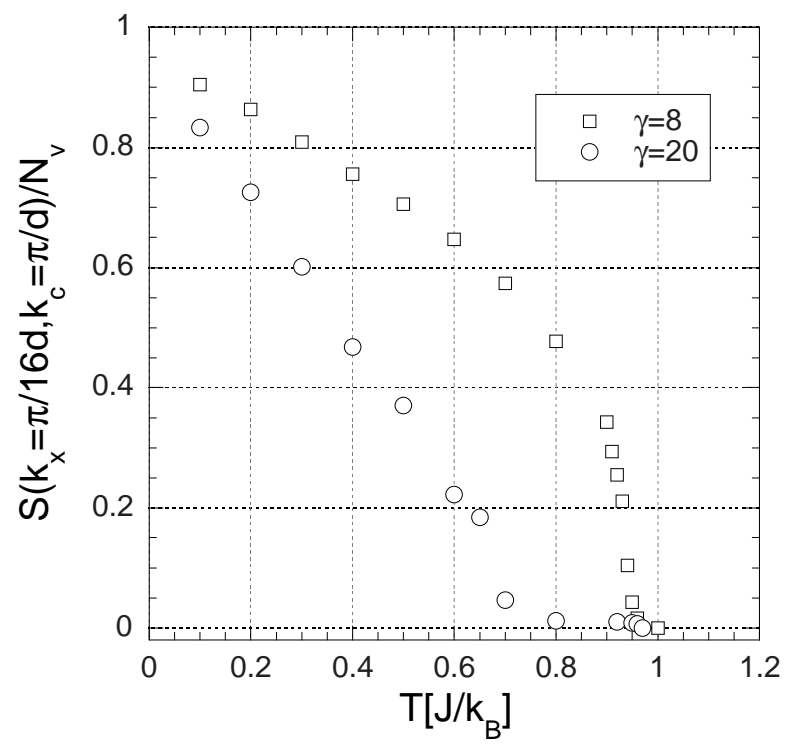

FIG. 7: Temperature dependence of the intensity of Bragg peaks for $\gamma=8$ and 20 .

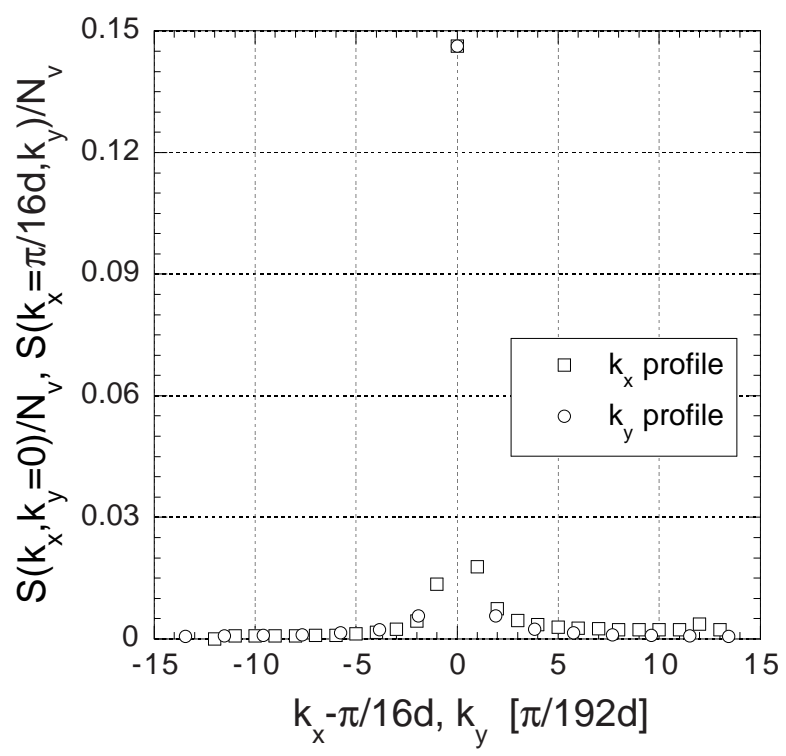

FIG. 8: Profiles of the structure factor $S\left(k_{x}, k_{y}\right)$ around the Bragg spot $\left(k_{x}, k_{y}\right)=(\pi / 16 d, 0)$ for $\gamma=20$ at $T=0.8 \mathrm{~J} / k_{B}$.

direction. Since $\eta<2$ observed in the structure factor $S\left(k_{x}, k_{c}\right)$, the unnormalized profiles should diverge in the thermodynamic limit as in the above equations. Therefore, one cannot evaluate the exponent $\eta$ directly from the profiles.

The above observations therefore indicate the existence of an intermediate phase for $\gamma=20$, characterized by SR interlayer correlation and in-plane QLRO, which is clearly absent for $\gamma=8$ below the multicritical point.

Is an intermediate phase plausible in the present system theoretically? According to the perturbative RG expansion to a 2D system by Balents and Nelson [25], 


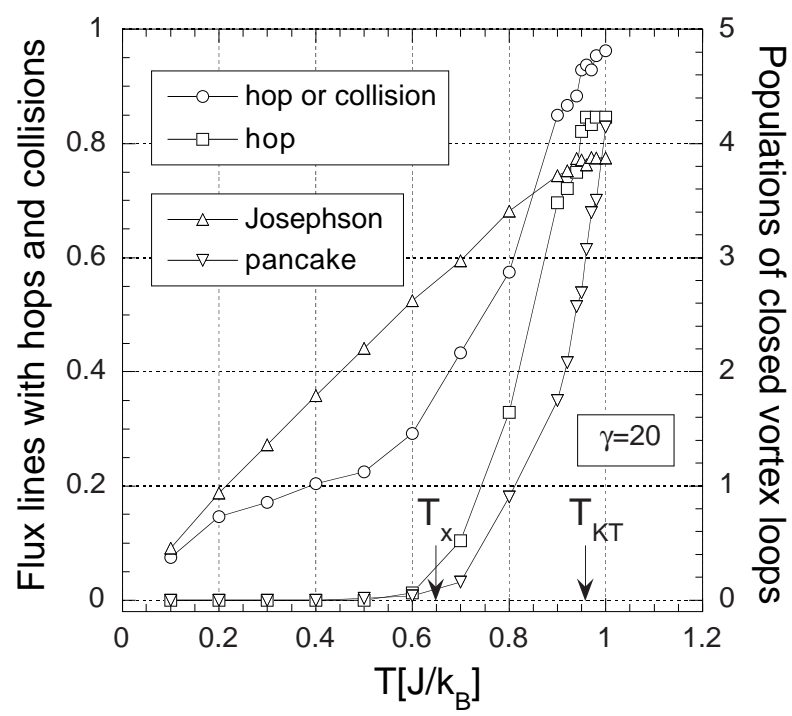

FIG. 9: Temperature dependence of ratios of Josephson flux lines which contain segments hopping into neighboring block layers, of those which either hop or collide with neighbors in the same block layers, and of populations of thermally excited, closed loops of Josephon vortices (normalized by $40 \times 240$ ) and of those containing also pancake vortices (normalized by 240).

the lattice is stable at low temperatures with $\eta<1 / 2$ where dislocations caused by hops of segments of Josephson flux lines across the superconducting layers are irrelevant while the interlayer coupling is relevant; at high temperatures with $\eta>2$ where the dislocations are relevant while the interlayer coupling is irrelevant, the system behaves as liquid. In the intermediate temperature regime, both of them are relevant and the fate of the system is not very well controlled. The system may take one of the two states, or it can take the smectic order as the authors suggested.

In simulations, we analyze the trajectories of the Josephson flux lines when they travel through the sample. As depicted in Fig 9 Josephson flux-lines are completely confined by the $\mathrm{CuO}_{2}$ layers below $T_{\times}$. Without dislocations caused by the hops, the system is in lattice phase as shown in the top-left panel of Fig 3 consistent with the theory. Dislocations become popular in the system when temperature is elavated cross $T_{\times}$as shown in Fig 9] It is interesting to observe that the maximal value of the exponent $\eta$ in our simulations, $\eta \simeq 1.97$ at $T \simeq 0.96 \mathrm{~J} / k_{B}$, is close to the theoretical prediction $\eta_{c}=2$ for the phase boundary between liquid and the intermediate phase. However, we should notice that while the theory predicted relevance of interlayer coupling in the intermediate regime, our simulations indicate the SR $c$-axis correlation. The origin of this discrepancy is not clear at this moment. As pointed out in Ref. [10], one possibility is that although the long-wave-length displacements of Josephson flux lines in the neighboring layers are coupled, accumulation of disorders in a stack of layers leads to decoupling, namely SR $c$-axis correlation.
In Fig 9 we also display the temperature dependence of population of closed Josephson vortex loops activated thermally. These excitations have not been treated in the elastic theory, and are responsible for the temperature dependence of the exponents $\eta$. The elastic constants in the present system are therefore not simply dominated by entropy as discussed in the elastic theory. Our system is similar to the thermotropic smectics in this aspect [2]]. A counter example is found in the lyotropic lamellar phase of a multilayer fluid membrane system [44]. See [45] for a detailed discussion.

\section{E. Novel Kosterlitz-Thouless phase}

Since the density-density correlations are SR in the $c$ direction in the intermediate phase, the effective free energy is governed by the long-wave-length fluctuations given in the following density expression

$$
\rho(\mathbf{r})=\psi(\mathbf{r}) e^{-i \mathbf{k}_{0} \cdot \mathbf{r}}+\psi^{*}(\mathbf{r}) e^{i \mathbf{k}_{0} \cdot \mathbf{r}},
$$

with $\mathbf{k}_{0} \equiv\left(k_{x 0}, k_{y 0}\right)=(2 f \pi / d, 0)$ and

$$
\psi(\mathbf{r})=|\psi| e^{i 2 f \pi u_{x} / d}
$$

When a small amplitude $|\psi|$ is set up [25], presumably at $T \simeq 0.96 \mathrm{~J} / k_{B}$ as in Figs 6 and 7 the effective free energy is reduced to

$$
F=\int d^{2} r\left(\nabla u_{x}\right)^{2}
$$

Since the displacement field $u_{x}$ is continuous and of the modulus $d / f$, the above free energy is effectively the same as the Hamiltonian of the 2D XY model. The intermediate phase therefore falls onto the KT fixed line.

\section{SUPERCONDUCTIVITY TRANSITION}

So far we have concentrated on the crystalline order of Josephson vortices. It is also important to investigate the superconductivity in the system. Although the amplitude of the local order parameter of superconductivity is fixed in the Hamiltonian (1), the fate of macroscopic superconductivity is governed by the order of Josephson vortices. We measure the LR superconductivity by the helicity modulus 31, 32].

As shown in Fig[10 in-plane helicity moduli for $\gamma=8$ set up sharply at the melting point $T_{m} \simeq 0.96 \mathrm{~J} / k_{B}$, where 3D LRCO is realized. The finite helicity modulus $\Upsilon_{y}$ along the magnetic field indicates the breaking of $\mathrm{U}(1)$ gauge symmetry, and thus the appearance of LRO of superconductivity. The finite $\Upsilon_{x}$ is, on the other hand, the signature of the intrinsic pinning on Josephson vortices from the layer structure, since the helicity modulus 


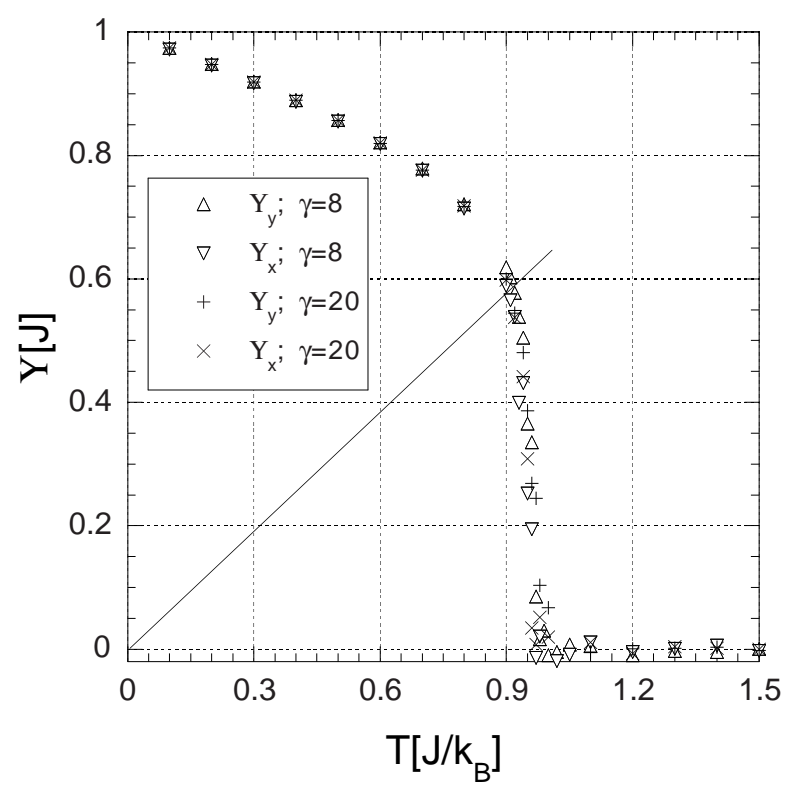

FIG. 10: Temperature dependence of in-plane helicity moduli for $\gamma=8$ and $\gamma=20$. The solid line is for theoretical prediction of the universal jump at KT transitions. For a pure 2D unfrustrated XY model corresponding to $\gamma=\infty$ in Hamiltonian (1) $T_{\mathrm{KT}}^{\mathrm{bare}} \simeq 0.89 \mathrm{~J} / k_{B}$.

$\Upsilon_{x}$ measures the energy cost for sliding Josephson vortices in the $c$ direction. $\Upsilon_{c}$ is vanishing down to zero temperature, reflecting the absence of pinning force in the $x$ direction. Therefore, the system is superconducting in $a b$ plane for $T \leq T_{m}$. The sharp drop of the in-plane helicity moduli at the melting point is in accordance with the first-order nature of the melting transition.

A typical configuration of phase variables on an $a b$ plane at low temperatures is displayed in Fig 11. The phases are uniform along the magnetic field. It is also regular in the $x$ direction, except for a single-wave-number modulation governed by the density of Josephson flux lines [11, 46].

The static phase wave can be displayed in a more transparent way by showing the supercurrent in the $x$ direction $I_{x}(\mathbf{r}) \sim \sin (\varphi(\mathbf{r}+\hat{\mathbf{x}})-\varphi(\mathbf{r}))$. The supercurrents associated with the phase distribution in Fig [11]are displayed in the left panel of Fig 12 Regions of positive and negative supercurrents in the $x$ direction are decorated by white and black tones respectively. There are four black (and white) stripes in the region of $l_{x}=128 d$, corresponding to the density of Josephson flux lines $f=1 / 32$. The pattern of supercurrents on a neighboring $a b$ plane is shown in the right panel of Fig 12 The stripes on the two planes are opposite to each other in black and white tones, and sustain four Josephson flux lines between them shown in Fig.2 of Ref. 37.

Variations of the phase variables along the $c$ direction is displayed in Fig [13] The configuration is similar to the sequence $(0,0, \pi, \pi, 0,0, \cdots)$ derived in Ref. 26. The simulated $c$-axis modulation is more complex, which to-

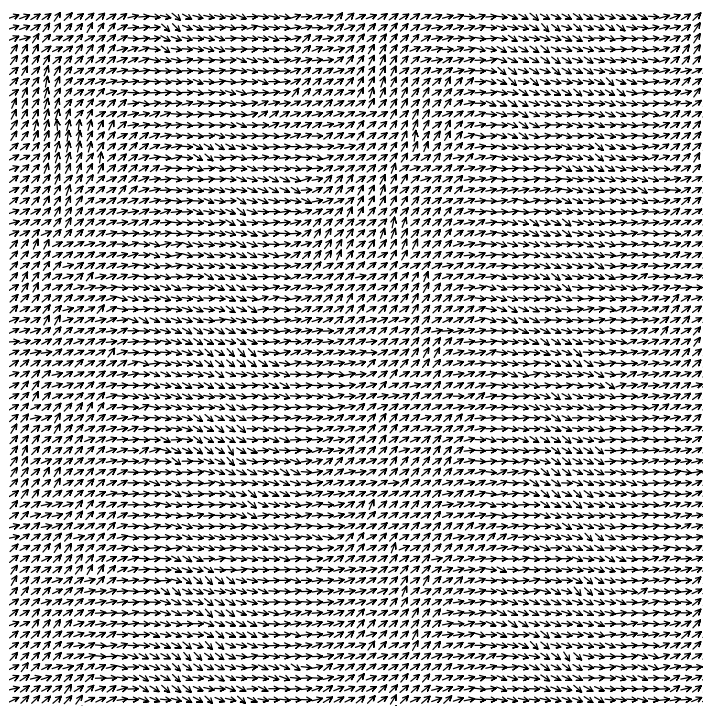

FIG. 11: A typical configuration of phase variables on an $a b$ plane at $T=0.1 J / k_{B}$ for $\gamma=8$. Shown is a region of dimensions $l_{x} \times l_{y}=64 d \times 64 d$.
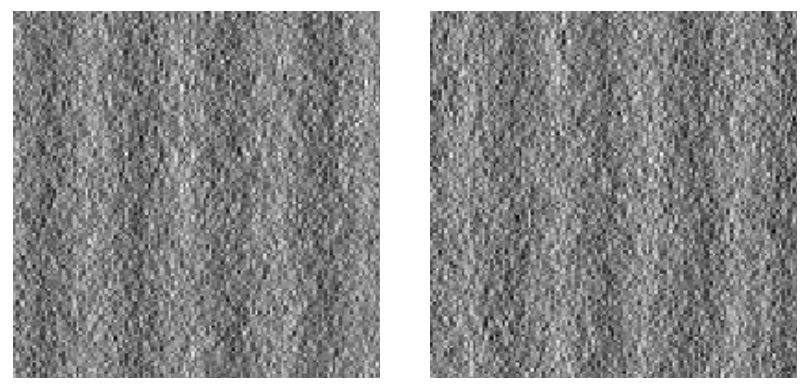

FIG. 12: Supercurrents between two sites neighboring to each other in the $x$ direction. Shown is the region of the same origin of Fig 11]but of larger dimensions $l_{x} \times l_{y}=128 d \times 128 d$.

gether with that in the $x$ direction minimizes the energy for weak but finite interlayer couplings.

The amplitude of the phase modulation in $x$ direction is small around and above the multicritical point in accordance with the analytic result by Bulaevsky and Clem: $\mathcal{A}=1 / 2 \pi(\gamma f)^{2}<1[46$. Most of the frustrations induced by the magnetic field are confined in the $c$ direction as clearly seen in Fig 13. The large phase modulations in the $c$ direction make the phase slip hard, resulting in the finite helicity modulus $\Upsilon_{x}$. The small, and thus soft phase modulations in the $x$ direction suppress the helicity modulus $\Upsilon_{c}$ to zero.

For $\gamma=20$, finite in-plane helicity moduli signal the onset of QLRO of superconductivity, as a twin of the QLRCO of Josephson vortices. The sharp drops of helicity moduli for $\gamma=20$ correspond to the universal jump in KT transitions [47].

The jump of helicity modulus in Fig 10 is rounded from the universal one by the finite-size effect as usual. An analysis on finite-size effects is certainly helpful here. 


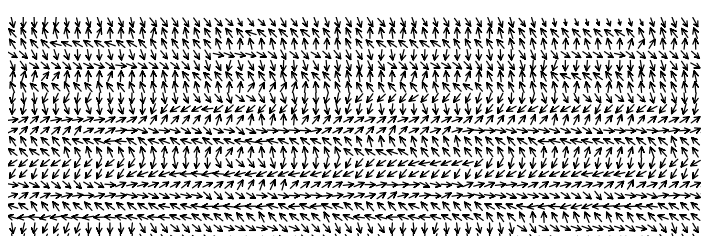

FIG. 13: A typical configuration of phase variables on an $a c$ section at low temperatures for $\gamma=8$. Shown is a region of dimensions $l_{x} \times l_{z}=64 d \times 20 d$.

However, as one can see in a careful study by Lee and Teitel on a 2D Coulomb gas system in Ref. 48], this approach is usually very hard since the finite-size effects are logarithmically weak 49]. A similar analysis for the present system should be even harder, since first, there is a SR correlation in the $c$ direction, secondly, the system size should be very huge in order to contain sufficient number of Josephson vortices, and thirdly, the present system is anisotropic in all three directions.

For these reasons, we will not seek to verify the universal jump in the present system. Instead, upon revealing the existence of the novel KT phase from simulation results and the symmetry argument in terms of the crystalline order of Josephson vortices in the preceding section, we adopt the expected universal jump 47

$$
\Upsilon / k_{B} T_{\mathrm{KT}}=2 / \pi
$$

to estimate the KT transition temperature [50]. From data in Fig 10] we find $T_{\mathrm{KT}} \simeq 0.91 \mathrm{~J} / k_{B}$.

The KT transition temperature for $\gamma=20$ is above that of the isolated $2 \mathrm{D}$ system $T_{\mathrm{KT}}^{\mathrm{bare}} \simeq 0.89 \mathrm{~J} / k_{B}$. This is physical since couplings between nearest neighboring layers enhance ordering. The bare KT transition point sets a lower bound for the normal to superconductivity transition in magnetic fields parallel to the $a b$ plane, as can be read from Hamiltonian (1).

An important feature in the helicity moduli for $\gamma=20$ is revealed in Fig 10 Namely, the helicity moduli in $x$ and $y$ directions are collapsing at all temperatures. Presuming that the relation between the exponent $a(T)$ defined in the I-V characteristics $V \sim I^{a(T)}$ and the helicity moduli [51, 52]

$$
a_{\mu}=1+\Upsilon_{\mu} \pi / k_{B} T, \quad \text { for } \quad \mu=x, y
$$

in the $\mathrm{KT}$ phase derived in pure $2 \mathrm{D}$ systems is also applicable for the present KT phase, the collapse of helicity moduli in the two in-plane directions is consistent with the orientation-independent, i.e. Lorentz-forceindependent, dissipations when the current and magnetic field are both parallel to the $a b$ plane [12]. Further investigation is expected in order to address this point completely.

One might notice that in Fig 10 the helicity moduli for the two anisotropy parameters are very close to each other. It is reasonable since, first, both first-order and KT transitions reveal sharp drops in the helicity modulus, and secondly, the anisotropy parameters are both very large such that the transition points are close to the lower bound $T_{\mathrm{KT}}^{\mathrm{b} a r e}=0.89 \mathrm{~J} / k_{B}$. The helicity moduli in the $x$ and $y$ directions are close to each other for $\gamma=8$ merely because that it is close to the multicritical point. For smaller anisotropy parameters, thus far from the multicritical point, we find clearly that the isotropy in the in-plane helicity modulus is broken. This explains the anisotropic resistivity in samples of $\mathrm{YBa}_{2} \mathrm{Cu}_{3} \mathrm{O}_{7-\delta}$.

\section{PHASE TRANSITION AT $T_{\mathrm{x}}$}

What is the nature of the phase transition between the 2D QLRO and 3D LRO at $T_{\times}$? In order to answer this question, we follow Balents and Nelson to compose an effective Landau free-energy functional for the 3D Josephson vortex lattice, which was formulated for a possible smectic to crystal transition [25]. The vortex density in the 3D crystal phase is expressed by three primitive reciprocal-lattice vectors as in Eq.(4). A Landau free energy for the dominant fluctuations is given by, up to a spatial anisotropy in the coefficients which is unimportant here,

$$
\begin{aligned}
F & =1 / 2 \int d^{3} R\left[h\left|\nabla \Psi_{1}\right|^{2}+h\left|\nabla \Psi_{2}\right|^{2}\right. \\
& +g\left(\nabla \Psi_{1} \nabla \Psi_{2}+\nabla \Psi_{1}^{*} \nabla \Psi_{2}^{*}\right) \\
& \left.+r\left(\left|\Psi_{1}\right|^{2}+\left|\Psi_{2}\right|^{2}\right)+w\left(\Psi_{1} \Psi_{2}+\Psi_{1}^{*} \Psi_{2}^{*}\right)+\cdots\right]
\end{aligned}
$$

where the order parameter $\Psi_{0}=1$ associated with $\left(k_{x}, k_{c}\right)=(0, \pm 2 \pi / d)$ has been included into the coefficients $g$ and $w$. Our simulations have revealed that Josephson flux lines are completely confined by superconducting layers, namely $u_{c}=0$, for $T<T_{\times}$. Thus, the two order parameters in Eq.(15) become complex conjugate to each other:

$$
\Psi_{1}=\Psi_{2}^{*}=|\Psi| e^{i 2 f \pi u_{x} / d} .
$$

The free energy is then reduced to

$$
F=\int d^{3} R\left(\nabla u_{x}\right)^{2}
$$

The phase transition is therefore second order with the critical phenomena governed by the universality class of the 3D XY model.

A phase transition in this universality class possesses a negative critical exponent $\alpha$ for the specific heat. This naturally explains the smooth temperature dependence of the simulated specific heat around $T_{\times}$in Fig. 3 of Ref. [37. 


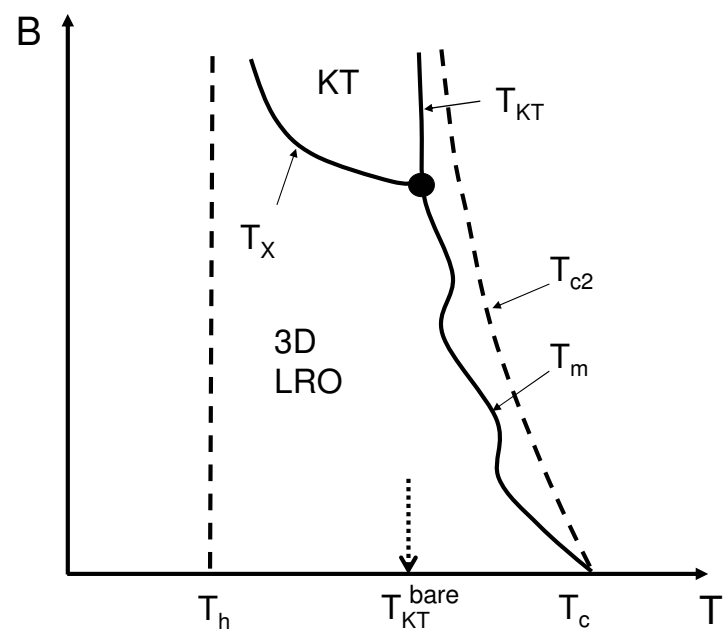

FIG. 14: $B-T$ phase diagram for interlayer Josephson vortices with a multicritical point at $B_{\mathrm{mc}}=\phi_{0} / 2 \sqrt{3} \gamma d^{2}$. The phase boundaries $T_{m}(B), T_{\mathrm{KT}}(B)$ and $T_{\times}(B)$ are associated with first-order, KT and 3D XY phase transitions as discussed in text. Possible crossovers are included by dashed lines.

When the critical point $T_{\times}$is approached from below, interlayer shear modulus of the Josephson vortex lattice is softened continuously to zero as $C_{66} \sim d / \xi_{c} \sim\left(T_{\times}-\right.$ $T)^{\nu}$ with $\nu \simeq 0.67$, and ramains vanishing in the whole intermediate phase.

\section{PHASE DIAGRAM}

Based on the analyses presented so long, we map out in Fig 14 the $B-T$ phase diagram for interlayer Josephson vortices, noting that the same physics should occur when the magnetic field is tuned at a given anisotropy parameter. The first-order melting line at low magnetic fields branches into two phase boundaries at the multicritical point characterized by the magnetic field

$$
B_{m c}=\frac{\phi_{0}}{2 \sqrt{3} \gamma d^{2}}
$$

The phase at high magnetic fields and intermediate temperatures is a novel KT phase.

There are two dashed, crossover curves in the phase diagram Fig 14. The high-temperature one denoted by $T_{c 2}$ follows the broad cusp in the specific heat where huge numbers of vortex loops are excited. It is a crossover where the amplitude of superconductivity order parameter attains a finite value, and thus corresponds to $H_{c 2}$ in the mean-field theory. The low-temperature and almostfield-independent line $T_{h}$ denotes a crossover temperature below which thermally activated pancake vortices are very few and bound to each other too tightly such that Josephson flux lines cannot hops across the $\mathrm{CuO}_{2}$ layers. Transport properties can be different on the two sides of the crossover line $T_{h}$ since randomly distributed point-like defects might pin Josephson flux lines via the pancake vortices present above $T_{h}$ [53, 54]. As the displacement in the $c$ direction is massive below $T_{h}$, one expects the transverse Meissner effect in the low temperature regime.

\section{SUMMARY AND DISCUSSIONS}

The main finding of the present work is that the normal to superconductivity phase transition in magnetic fields parallel to superconducting layers is first order at low fields, while two-step and continuous with a KT type intermediate phase at high fields. There is a multicritical point at magnetic field of order $B_{\mathrm{mc}}=\phi_{0} / 2 \sqrt{3} \gamma d^{2}$ in the $B-T$ phase diagram.

That the above phases are genuine for the interlayer Josephson vortices rather than artifacts from finite-size and short-time effects can be concluded from the following considerations: First, the successful observation of the single, first-order melting transition for $\gamma=8$ indicates that the system size is sufficient for the onset of 3D LRCO below the multicritical anisotropy parameter; Second, the SR order in the $c$ direction for $\gamma=20$ is not a finite-size effect, since periodic boundary conditions are adopted in the present study, which tend to enhance ordering. It is not a short simulation-time effect either, since a heating process from a $3 \mathrm{D}$ LR crystalline order is adopted; Third, it is clear that the system described by Hamiltonian (1) with a finite $\gamma$ should order (maybe partially) at a temperature higher than that of the limit case $\gamma=\infty$, which composes of independent 2D superconductors with $T_{\mathrm{KT}}^{\mathrm{bare}} \simeq 0.89 \mathrm{~J} / k_{B}$. Thus, the in-plane vortex correlation functions at, for example, $T=0.7$ and $0.8 \mathrm{~J} / k_{B}<T_{\mathrm{KT}}^{\mathrm{bare}}$ for $\gamma=20$ cannot be SR; with the short rangeness of $c$-axis correlations, they cannot be LR either. They then should be QLR, consistent with that demonstrated numerically.

The peculiar transport properties observed in $\mathrm{Bi}_{2} \mathrm{Sr}_{2} \mathrm{CaCu}_{2} \mathrm{O}_{8+y}$ when magnetic fields and currents are both parallel to the $\mathrm{CuO}_{2}$ layers are able to be explained in terms of the $B-T$ phase diagram, since dissipation at small currents should be governed by the equilibrium properties. In the KT phase, isotropic helicity moduli with the universal jump at the transition point are responsible to the isotropic, and therefore Lorentz-force-independent, dissipation and the power-law non-Ohmic I-V characteristics. In the 3D lattice phase, which is realized at low magnetic fields for Bi-based materials or up to quite strong fields in YBCO materials, larger dissipations should be observed at transverse currents than parallel ones.

The $B-T$ phase diagram in Fig [14 is consistent with several recent experiments. By detecting the oscillation in the $c$-axis flux flow of Josephson vortices in $\mathrm{Bi}_{2} \mathrm{Sr}_{2} \mathrm{CaCu}_{2} \mathrm{O}_{8+y}$, Ooi and Hirata succeeded in measuring the phase boundary on which the 3D triangular lattice softens [55]. The resultant curve has the same 
shape formed by $T_{\times}(B)$ at high fields and $T_{m}(B)$ at low fields, with a kink corresponding to the multicritical point. In the same material, Mirkovic et al. observed that the sharp drop of the resistivity associated with the first-order vortex lattice melting at low magnetic fields is suppressed into a smooth one when the magnetic field is elevated to about 1T, suggesting a continuous phase transition [56]. The steep normal to superconductivity phase boundary at high magnetic fields observed by Lundqvist et al. [57] is in accordance with the lower bound $T_{\mathrm{KT}}^{\mathrm{bare}}$ on $T_{\mathrm{KT}}(B)$, although the KT features are still not available experimentally.

Recently Kakeya et al. observed two plasma modes when applying a magnetic field parallel to the $a b$ plane, of frequency higher (lower) than that of zero magnetic field and increasing (decreasing) with the magnetic field 58. While the high branch is caused by the resonance between the electromagnetic field and coherent motion of interlayer Josephson vortices, the low branch is asigned to the shear vibration of Josephson vortex lattice [59]. Since the KT phase proposed in the present work is characterized by vanishing interlayer shear modulus, the low plasma mode should disappear and the high mode is still observable as the magnetic field or temperature approach the phase boundary $T_{\times}$from the $3 \mathrm{D}$ lattice phase. Therefore, the Josephson plasma phenomenon provides a powerful technique to test our phase diagram.

Since the multicritical field is approximately 50T for $\mathrm{YBa}_{2} \mathrm{Cu}_{3} \mathrm{O}_{7-\delta}$ presuming $\gamma=8$ and $d=12 \AA$, the normal to superconductivity phase transition should be first order at magnetic fields available in laboratory to date. This is consistent with a recent measurement on the specific heat by Schilling et al. up to $10 \mathrm{~T}$ [60]. It is revealed that the phase boundary is smooth in accordance with the 3D anisotropic GL theory, and thus the system is essentially the same as the Abrikosov (or pancake) vortex system. A meandering phase boundary was observed in transport measurements by Gordeev et al. in the same family of materials and field regime 54]. The difference might be quantitative, or may be the result of different experimental techniques. It is noticed that our phase diagram is not to scale at low fields.

It is interesting to ask whether the phase diagram of interlayer Josephson vortices can be derived from a Lindemann type theory. In a Lindemann theory a lattice is supposed to melt when the thermal average of displacement field exceeds a certain fraction of inter-vortex distance. This picture has been useful in understanding the melting of pancake vortex lattice (or Bragg glass). One should be very careful when applying the Lindemann the- ory to the melting of interlayer Josephson vortex lattice, since thermal fluctuations are very anisotropic especially at strong magnetic fields above the multicritical point. While thermal fluctuations in the $c$ direction are pinned completely below $T_{\times}$, those in $a b$ planes diverge as temperature approaches it from below. Layers of Josephson vortices slide easily over each other in the intermediate phase. A naive Lindemann theory is clearly not applicable.

It is important to notice the differences among the present system and smectic liquid crystals with or without an external field. Ideal smecitc-A liquid crystals have liquid-like correlations in two dimensions and a solid like periodic modulation of the density along the third direction. The elastic free-energy functional for a smectic possesses the so-called Landau-Peierls instability 61, 62, 63], i.e. suppression of in-plane quadratic first derivatives. When a magnetic or dielectric field is applied, the layer normal is energetically confined in a predetermined plane. The quadratic first derivative is then suppressed in only one direction. Systems in this group were called planar layered, and the possible phases and phase diagrams were addressed by Grinstein et al. 64]. The interlayer Josephson-vortex system in magnetic fields parallel to the layers is polar layered, in which the normal of Josephson-vortex layers is along the crystalgraphic $c$ axis. There is no Landau-Peierls like instability left any more in the present system. The difference in symmetry results in the different phases, as clearly indicated by the simulated structure factors. It is interesting to observe the similarity between the last two layered systems, namely both of them contain a multicritical point in their phase diagrams.

\section{Acknowledgments}

The author would like to thank L. Balents, G. Baskaran, G. Blatter, L. Bulaevskii, Q.-H. Chen, J. Clem, G. Crabtree, K. Hirata, T. Ishida, K. Kadowaki, A. Koshelev, W. Kwok, S. Miyashita, D. Nelson, Y. Nonomura, A. Tanaka, V. Vinokur, U. Welp, and A. Zhukov for stimulating discussions. Simulations are performed on the Numerical Materials Simulator (SX-5) of National Institute for Materials Science, Japan. This work was partially supported by Japan Society for the Promotion of Science (Grant-in-Aid for Scientific Research (C) No. $15540355)$.
[1] S. Kamal, D. A. Bonn, N. Goldenfeld, P. J. Hirschfeld, R. X. Liang, and W. N. Hardy, Phys. Rev. Lett. 73, 1845 (1994).

[2] A. A. Abrikosov, Zh. Eksp. Teor. Fiz. 32, 1442 (1957) [Phys. JETP 5, 1174 (1957)].
[3] G. Blatter, M. V. Feigel'man, V. B. Geshkenbein, A. I. Larkin, and V. M. Vinokur, Rev. Mod. Phys. 66, 1125 (1994).

[4] G. W. Crabtree, and D. R. Nelson, Physics Today 45, 38 (1997). 
[5] T. Nattermann and S. Scheidl, Advances in Physics 49, 607 (2000).

[6] G. J. Dolan, F. Holtzberg, C. Feild, and T. R. Dinger, Phys. Rev. Lett. 62, 2184 (1989).

[7] M. Tachiki and S. Takahashi, Solid State Commun. 70, 291 (1989).

[8] K. B. Efetov, Sov. Phys. JETP 49, 905 (1979).

[9] B. I. Ivlev, N. B. Kopnin and V. L. Pokrovsky, J. Low Temp. Phys. 80, 187 (1990); B. I. Ivlev, N. B. Kopnin and M. M. Slomaa, Phys. Rev. B 43, 2896 (1991).

[10] L. V. Mikheev and E. B. Kolomeisky, Phys. Rev. B 43, 10431 (1991).

[11] S. E. Korshunov and A. I. Larkin, Phys. Rev. B 46, 6395 (1992).

[12] Y. Iye, S. Nakamura, and T. Tamegai, Physica 159C, 433 (1989).

[13] Y. Ando, N. Motohira, K. Kitazawa, J. Takeya, and S. Akita, Phys. Rev. Lett. 67, 2737 (1991).

[14] A. K. Pradhan, S. J. Hazell, J. W. Hodby, C. Chen, Y. Hu, and B. M. Wanklyn, Phys. Rev. B 47, 11374 (1993).

[15] S. Chakravarty, B. I. Ivlev, and Y. N. Ovchinnikov, Phys. Rev. Lett. 64, 3187 (1990).

[16] V. L. Berezinsky, Sov. Phys. JETP 34, 610 (1972).

[17] J. M. Kosterlitz and D. J. Thouless, J. Phys. C 6, 1181 (1973).

[18] G. Blatter, B. I. Ivlev, and J. Rhyner, Phys. Rev. Lett. 66, 2392 (1991).

[19] B. Horovitz, Phys. Rev. B 47, 5947 (1993); ibid 5964 (1993).

[20] T. T. M. Palstra, B. Batlogg, L. F. Schneemeyer, and J. V. Waszczak, Phys. Rev. Lett. 61, 1662 (1988).

[21] W. K. Kwok, U. Welp, G. W. Crabtree, K. G. Vandervoort, R. Hulscher, and J. Z. Liu, Phys. Rev. Lett. 64, 966 (1990).

[22] R. C. Budhani, D. O. Welch, M. Suenaga, and R. L. Aabatini, Phys. Rev. Lett. 64, 1666 (1990).

[23] P. H. Kes, J. Aarts, V. M. Vinokur, and C. J. van der Beek, Phys. Rev. Lett. 64, 1063 (1990).

[24] W. K. Kwok, J. Fendrich, U. Welp, S. Fleshler, J. Downey, and G. W. Crabtree, Phys. Rev. Lett. 72, 1088 (1994).

[25] L. Balents and D. R. Nelson, Phys. Rev. Lett. 73, 2618 (1994); ibid Phys. Rev. B 52, 12951 (1995).

[26] L. Balents and L. Radzihovsky, Phys. Rev. Lett. 76, 3416 (1996).

[27] M. P. A. Fisher and D. H. Lee, Phys. Rev. B 39, 2756 (1989).

[28] L. I. Glazman and A. E. Koshelev, Phys. Rev. B 43, 2835 (1991).

[29] E. Frey, D. R. Nelson and D. S. Fisher, Phys. Rev. B 49, 9723 (1994).

[30] R. E. Hetzel, A. Sudbo, and D. A. Huse, Phys. Rev. Lett. 69, 518 (1992).

[31] Y.-H Li and S. Teitel, Phys. Rev. B 47, 359 (1993); ibid 49, 4136 (1994).

[32] X. Hu, S. Miyashita, and M. Tachiki, Phys. Rev. Lett. 79, 3498 (1997); ibid Phys. Rev. B 58, 3438 (1998).

[33] A. Koshelev, Phys. Rev. B 56, 11201 (1997).

[34] A. K. Nguyen and A. Sudbo, Phys. Rev. B 58, 2802 (1998).

[35] P. Olsson and S. Teitel, Phys. Rev. Lett. 82, 2183 (1999).
[36] X. Hu and M. Tachiki, Phys. Rev. Lett. 80, 4044 (1998).

[37] X. Hu and M. Tachiki, Phys. Rev. Lett. 85, 2577 (2000).

[38] W. E. Lawrence and S. Doniach, in Proceedings of LT12, Tokyo, 1970, edited by E. Kanda (Keigaku, Tokyo, 1971), p.361.

[39] M. Dodgson, V. B. Geshkenbein, H. Nordborg, and G. Blatter1, Phys. Rev. Lett. 80, 837 (1998).

[40] P. Olsson and P. Holme, Phys. Rev. Lett. 85, 2651 (2000).

[41] L. D. Landau and E. M. Lifshitz, Statitical Physics Part 1 (Pergamon, 1980).

[42] J. Als-Nielsen, J. D. Litster, R. J. Birgeneau, M. Kaplan, C. R. Safinya, A. Lindegaard-Andersen, and S. Mathiesen, Phys. Rev. B 22, 312 (1980).

[43] G. Blatter, V. B. Geshkenbein, and A. I. Larkin, Phys. Rev. Lett. 68, 875 (1992).

[44] C. R. Safinya, D. Roux, G. S. Smith, S. K. Sinha, P. Dimon, N. A. Clark, and A. M. Bellocq, Phys. Rev. Lett. $\mathbf{5 7}, 2718$ (1986).

[45] P. M. Chaikin and T. C. Lubensky, Principles of condensed matter physics (Cambridge University Press, 1995).

[46] L. Bulaevskii and J. R. Clem, Phys. Rev. B 44, 10234 (1991).

[47] D. R. Nelson and J. M. Kosterlitz, Phys. Rev. Lett. 39, 1201 (1977); see also J. M. Kosterlitz, J. Phys. C 7, 1046 (1974).

[48] J.-R. Lee and S. Teitel, Phys. Rev. B 46, 3247 (1992).

[49] H. Weber and P. Minnhagen, Phys. Rev. B 37, 5986 (1988).

[50] P. Minnhagen, Rev. Mod. Phys. 59, 1001 (1987).

[51] V. Ambegaokar, B. I. Halperin, D. R. Nelson and E.D. Siggia, Phys. Rev. Lett. 40, 783 (1978); Phys. Rev. B 21, 1806 (1980).

[52] Q. H. Chen, L. H. Tang, and P. Q. Tong, Phys. Rev. Lett. 87, 067001(2001); L. H. Tang and Q. H. Chen, Phys. Rev. B 67, 024508 (2003).

[53] K. Hirata, private commnunication.

[54] S. N. Gordeev, A. A. Zhukov, P. A. J. de Groot, A. G. M. Jansen, R. Gagnon, and L. Taillefer, Phys. Rev. Lett. 85, 4594 (2000).

[55] S. Ooi and K. Hirata, Phys. Rev. Lett. 89, 247002 (2002).

[56] J. Mirkovic, S. E. Savel'ev, E. Sugahara, and K. Kadowaki, Phys. Rev. Lett. 86, 886 (2001).

[57] B. Lundqvist, O. Rapp, and M. Andersson, Phys. Rev. B 62, 3542 (2000); B. Lundqvist, O. Rapp, M. Andersson, and Y. Eltsev, 64, R060503 (2001).

[58] I. Kakeya, T. Wada, K. Kadowaki and M. Machida, Physica C 378-381, 437 (2002).

[59] T. Koyama, Phys. Rev. B 68, 224505 (2003); preprint.

[60] A. Schilling, U. Welp, W. K. Kwok, and G. W. Crabtree, Phys. Rev. B 65, 054505 (2002).

[61] L. D. Landau, in Collected Papers of L. D. Landau, edited by D. Ter Haar (Gordon and Breach, New York, 1965), p. 209.

[62] R. E. Peierls, Helv. Phys. Acta. 7, Suppl., 81(1934).

[63] P. G. de Gennes and J. Prost, The Physics of Liquid Crystals (Clarendon, Oxford, 1993).

[64] G. Grinstein, T. C. Lubensky, and J. Toner, Phys. Rev. B 33, 3306 (1986). 